
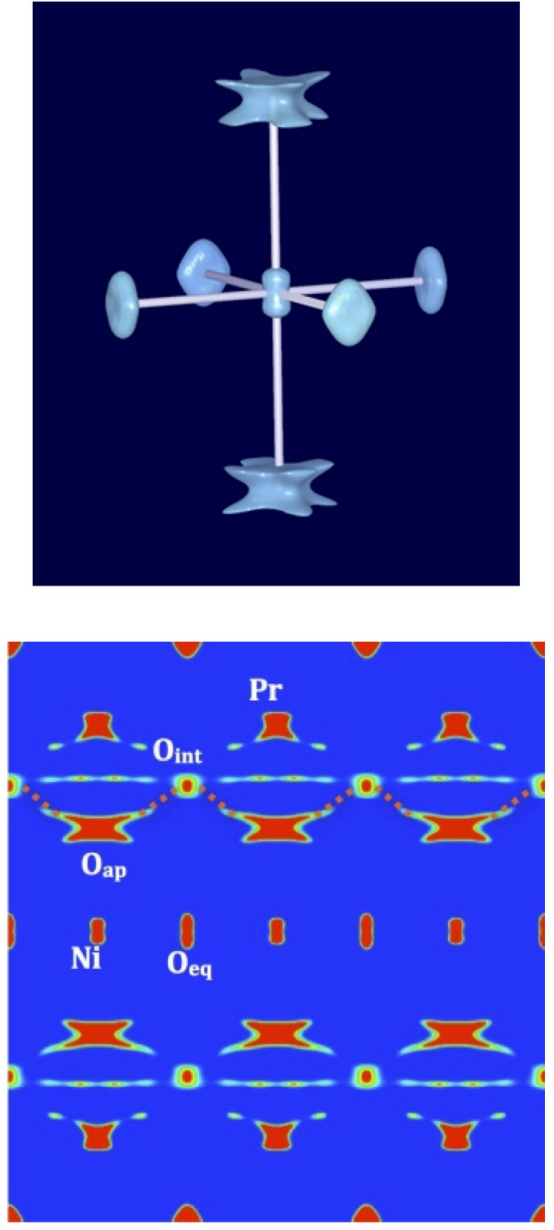

Figure 1. Nuclear density of $\mathrm{Pr}_{2} \mathrm{NiO}_{425} @ 400^{\circ} \mathrm{C}$ from single crystal neutron diffraction and subsequent MaxEnt reconstruction. Top: Anharmonic double potential of the $\mathrm{O}$ atoms of the $\mathrm{NiO}$ octahedra. Bottom: Dynamically activated oxygen diffusion pathway in the $\mathrm{Pr}_{2} \mathrm{O}_{2}$ rock salt layer between $\mathrm{O}_{\text {int }}$ and $\mathrm{O}_{\text {ap }}$ sites[3]

Keywords: transition metal oxides, phonon assisted diffusion, oxygen intercalation, in situ diffraction, inelastic neutron scattering, SOFC

\section{KN-14 Colour X-ray Tomography}

\author{
Bob Cernik ${ }^{1}$
}

1. School of Materials, The University of Manchester, UK

email: B.Cernik@manchester.ac.uk

The twentieth century saw a revolution in the field of $\mathrm{X}$-ray imaging beginning with the acquisition of somewhat rudimentary $2 \mathrm{D}$ radiographs and finishing with the arrival of advanced CT systems capable of producing very high quality $3 \mathrm{D}$ images that can show the internal structure of an object. The most common uses of CT can be found in non-destructive testing; security scanning and in hospitals where modern spiral CT scanners can collect whole body images in minutes. CT works on the basic principle that an X-ray beam incident on an object is absorbed by differing amounts depending upon the composition of the material and the shape of the object. By measuring the transmitted X-rays we obtain 2D radiographs. If we then rotate or translate the sample a number of radiographs can be collected from which 3D, density contrast, images can be mathematically reconstructed. These images can be made sharper by the use of phase or diffraction contrast but are still dominated by absorptive processes. The main limitations of radiography and CT are that they lack insight into the chemical, atomic or crystallographic structure of the object under study. This information can be crucial to fully understanding in-situ chemical processes. Examples of this can be found in corrosion mechanics; alloying behaviour or catalytic reactions. This talk is targeted toward the development and use of bright and dark field colour X-ray imaging systems capable of forming 3D images of relatively large objects using high power, high energy, X-ray sources (from lab and synchrotron). The colour images generated have chemical or physical information in each voxel of the image. Two main approaches will be discussed using high energy monochromatic and white or pink beam radiation. The monochromatic approach is usually called XRD-CT and can be configured to deliver excellent spatial resolution. XRD-CT has recently been demonstrated to work well with PDF data delivering nanocrystalline maps. The energy dispersive methods are faster but have poorer spatial and energy resolution but are more likely to be adopted for non-destructive testing and security scanning applications. EDD imaging has been facilitated by the development of pixelated energy sensitive X-ray detectors and by the development of scattering geometries to improve the signal to noise ratio (SNR) of the weak signals that contain this chemical and structural information.

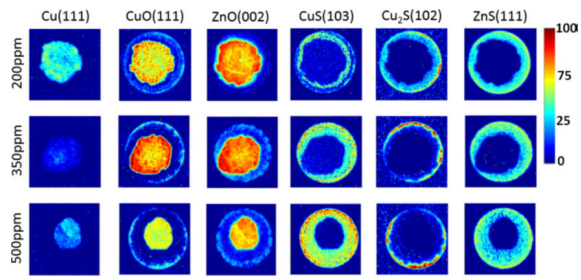

Figure 1. The figure shows how the crystalline phases within a $3 \mathrm{D}$ catalyst body $\left(\mathrm{Cu} / \mathrm{ZnO} / \mathrm{Al}_{2} \mathrm{O}_{3}\right)$ change after various levels of $\mathrm{H}_{2} \mathrm{~S}$ exposure. The 2D reconstructions were based on XRD-CT principles. Beale A $\mathrm{M}$ et al. JOURNAL OF CATALYSIS Vol 314, 94-100 (2014) 


\section{KN-15 Self-assembly of Nanoparticles Through Hydrophobic Interactions}

Marek Grzelczak ${ }^{1,2}$

1. CIC biomaGUNE, San Sebastian, Spain

2. Ikerbasque, Basque Foundation for Science, Spain

email:mgrzelczak@cicbiomagune.es

Bottom-up self-assembly of nanoscale objects is a central premise of nanotechnology. Besides tremendous progress in last decades, the liquid-phase self-assembly of nanoparticles remains theoretically and experimentally challenging especially when dealing with non-specific interactions. We exploit hydrophobic interactions to show that polystyrene (PS)-stabilized nanoparticles dispersed in tetrahydrofuran (THF) aggregate upon addition of water to form a variety of complex architectures (Figure 1$).^{[1-6]}$ The final length-scale, interparticle distances, and shape of the aggregates are driven by nature of the surface chemistry, the geometry of particles building blocks, or rate of water addition. Since the integrity of the assemblies is maintained by non-covalent interactions, the presence of external stimuli induces disintegration, internal rearrangement or even division of the clusters that makes them an attractive material for (bio)sensing.

\section{References}

[1] M. Grzelczak, A. Sánchez-Iglesias, H. H. Mezerji, S. Bals, J. Pérez-Juste, L. M. Liz-Marzán, Nano Lett. 2012, 12, 4380.

[2] A. Sánchez-Iglesias, M. Grzelczak, T. Altantzis, B. Goris, J. Pérez-Juste, S. Bals, G. Van Tendeloo, S. H. Donaldson, B. F. Chmelka, J. N. Israelachvili, L. M. Liz-Marzán, ACS Nano 2012, 6, 11059.

[3] M. Grzelczak, A. Sánchez-Iglesias, L. M. Liz-Marzán, Soft Matter 2013, 9, 9094

[4] J. E. Galván-Moya, T. Altantzis, K. Nelissen, F. M. Peeters, M. Grzelczak, L. M. Liz-Marzán, S. Bals, G. Van Tendeloo, ACS Nano 2014, 8, 3869.

[5] M. Grzelczak, A. Sánchez-Iglesias, L. M. Liz-Marzán, CrystEngComm 2014, 16, 9425.

[6] A. L. Porta, A. Sánchez-Iglesias, T. Altantzis, S. Bals, M. Grzelczak, L. M. Liz-Marzán, Nanoscale 2015, 7, 10377.

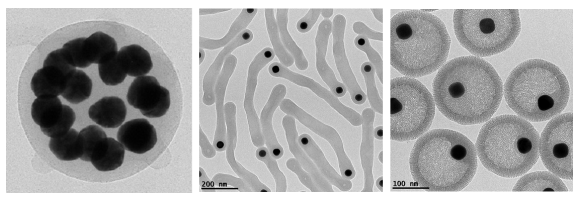

Figure 1. Solvent-induced self-assembly of polystyrene-coated gold nanoparticles. (left) Spherical clusters consisting of gold nanoparticles with $20 \mathrm{~nm}$ of diameter. The interparticle gap is controlled by the length of the polymer chains grafted on the particles surface. (middle) Eel-like polymeric structure containing metallic nanoparticles on terminus. (right) Hollow mesoporous silica capsules carrying hydrophobic nanoparticles coated with polystyrene.

Keywords: Self-assembly of nanoparticles, hydrophobic interactions 